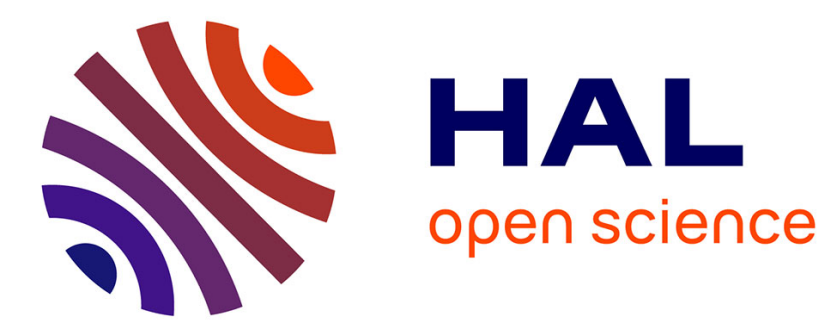

\title{
Planar non-linear free vibrations of an elastic cable
} Angelo Luongo, Giuseppe Rega, Fabrizio Vestroni

\section{To cite this version:}

Angelo Luongo, Giuseppe Rega, Fabrizio Vestroni. Planar non-linear free vibrations of an elastic cable. International Journal of Non-Linear Mechanics, 1984, 19 (1), pp.39-52. hal-00787648

\section{HAL Id: hal-00787648 \\ https://hal.science/hal-00787648}

Submitted on 12 Feb 2013

HAL is a multi-disciplinary open access archive for the deposit and dissemination of scientific research documents, whether they are published or not. The documents may come from teaching and research institutions in France or abroad, or from public or private research centers.
L'archive ouverte pluridisciplinaire HAL, est destinée au dépôt et à la diffusion de documents scientifiques de niveau recherche, publiés ou non, émanant des établissements d'enseignement et de recherche français ou étrangers, des laboratoires publics ou privés. 


\title{
PLANAR NON-LINEAR FREE VIBRATIONS OF AN ELASTIC CABLE
}

\author{
A. LUONGO*, G. REGA† and F. VESTRONI*
}

* Istituto di Scienza delle Costruzioni, Università di Roma Via Eudossiana 18, 00184 Roma, Italy

†Istituto di Scienza delle Costruzioni, Universitả dell'Aqquila Monteluco-Roio, 67100 L'Aquila, Italy

\begin{abstract}
Continuum non-linear equations of free motion of a heavy elastic cable about a deformed initial configuration are developed. Referring to an assumed mode technique one ordinary equation for the cable planar motion is obtained via a Galerkin procedure, an approximate solution of which is pursued through a perturbation method. Suitable nondimensional results are presented for the vibrations in the first symmetric mode with different values of the cable properties. Which procedure is the proper one to account consistently for the non-linear kinematical relations of the cable in one ordinary equation of motion is discussed.
\end{abstract}

\section{INTRODUCTION}

Some works have recently appeared in the literature concerning non-linear oscillations of curved structural elements. The occurrence of the initial curvature gives rise to both quadratic and cubic terms in the relevant equations of motion and this strongly influences the behaviour of these elements at large amplitudes. Most of the studies refer to curved beams, rings and shallow arches [1-5].

As far as suspended cables are concerned, little work has appeared on the matter of nonlinear free and forced oscillations, regarding both the theoretical formulation of the problem and the analysis of the structural behaviour [6-10]. Nevertheless finite vibrations of elastic cables are interesting for technical applications and some aspects in the relevant non-linear theory are still to be investigated.

In the present work non-linear equations of motion of a heavy elastic cable about a deformed static equilibrium configuration are developed through a Lagrangian description of the continuum problem in the context of dynamics of initially stressed solids [11]. After introducing consistent simplifying assumptions, the partial differential equations of planar motion are reduced to one ordinary equation via a Galerkin procedure by assuming a modal deflection shape. An approximate solution is pursued through the method of Lindstedt-Poincare by retaining terms up to the fourth order of the perturbation parameter; the frequency-amplitude relationship and the law of the motion are then obtained in closed form.

Numerical results are presented for the vibrations of a cable in the first symmetric in-plane mode in the range of technical values of its properties, showing that the retention of higher terms in the asymptotic expansion allows one to describe correctly the frequency-amplitude relationship.

Within this work attention is focused on two aspects of the formulation of the problem. The possibility of introducing a dimensionless form suitable to describe the non-linear behaviour of a cable in terms of a unique parameter characterizing its mechanical and geometrical properties, as it occurs in the linearized oscillations [12,13], is analysed. Also, which approximate procedure is the proper one to obtain one ordinary equation of motion that accounts consistently for the non-linear kinematical relations of the system is discussed.

\section{FORMULATION OF THE CONTINUUM PROBLEM}

Consider a heavy hyperelastic cable suspended between two fixed supports; let $O x_{i}$ be an orthogonal coordinate system which is referred to in the description of the cable configurations. Three of these are distinguished (Fig. 1): (a) the natural configuration $\mathscr{C}^{\circ}$ in which the material point $P$ has the location $P^{\circ}\left(s^{\circ}\right), s^{\circ}$ being a curvilinear abscissa, the length of the cable is $l_{c}^{\circ}$, the mass per unit length $m^{\circ}$, the area of normal section $A$, the elastic material 


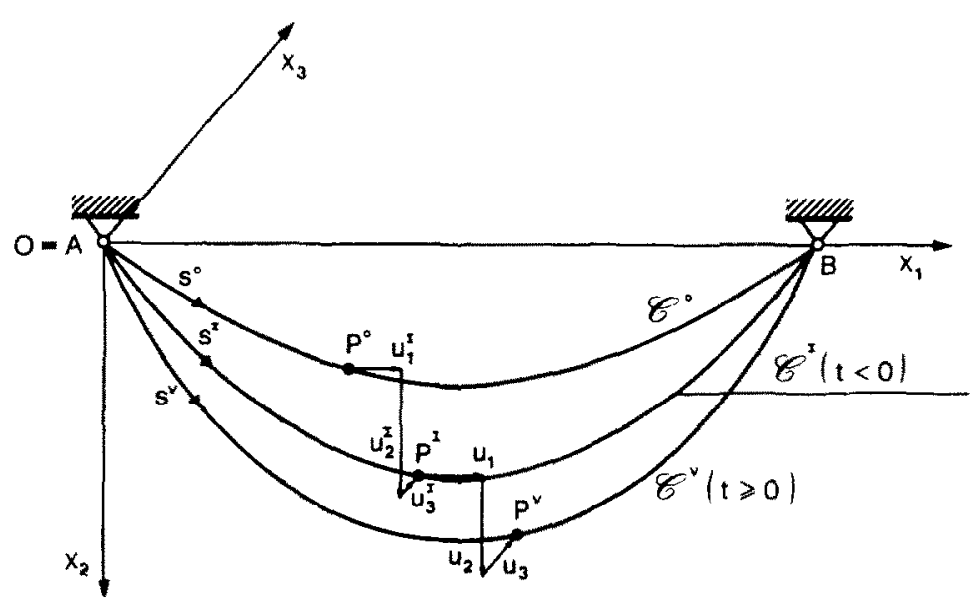

Fig. 1. Cable configurations.

modulus $E$; stress, strain and strain energy density vanish identically in $\mathscr{C}^{\circ}$; (b) the initial deformed configuration $\mathscr{C}^{I}$ of static equilibrium under dead load, in which $\varepsilon^{I}$ is the Lagrangian strain, $T^{I}$ the cable tension and $\phi^{I}$ the strain energy density; (c) the varied configuration $\mathscr{C}^{V}$ occupied by the cable during the motion, $\varepsilon^{V}, T^{V}$ and $\phi^{V}$ being the relevant quantities.

The initial deformation carries the material point $P$ from the location $P^{\circ}\left(x_{i}^{\circ}\right)$ to the location $P^{I}\left(x_{i}^{I}\right)$; the additional deformation carries it to its final location $P^{V}\left(x_{i}^{V}\right)$. By assuming $\mathscr{C}^{I}$ as the reference configuration for the cable motion, the components $u_{i}$ of the dynamic displacement field are given by

$$
u_{i}\left(s^{I}, t\right)=x_{i}^{Y}\left(s^{l}, t\right)-x_{i}^{I}\left(s^{I}\right)
$$

The Lagrangian strain is assumed as the strain measure; the total strain in $\mathscr{C}^{v}$ is

$$
\varepsilon^{V}=\frac{1}{2}\left(\frac{\partial x_{i}^{V}}{\partial s^{0}} \frac{\partial x_{i}^{V}}{\partial s^{0}}-1\right)
$$

which accounting for equation (1) can be written

$$
\varepsilon^{V}=\varepsilon^{I}+\varepsilon\left(\mathrm{d} s^{I} / \mathrm{ds}^{\circ}\right)^{2}
$$

where $\varepsilon^{I}$ is the initial Lagrangian strain and

$$
\varepsilon=\frac{\partial u_{i}}{\partial s^{l}} \frac{\partial x_{i}}{\hat{c} s^{I}}+\frac{1}{2} \frac{\partial u_{i}}{\partial s^{l}} \frac{\partial u_{i}}{\partial s^{I}}
$$

is the additional strain referred to the initial state.

It is assumed that the strain energy density per unit length of the natural state $\phi\left(\varepsilon^{f}\right)$ exists and is continuous in $\mathscr{C}^{I}$ together with its derivatives up to the third order. The strain energy density in $\mathscr{C}^{V}$ is expressed through a quadratic function of the additional strain:

$$
\phi\left(\varepsilon^{V}\right)=\phi\left(\varepsilon^{I}\right)+\left(\frac{\mathrm{d} \phi}{\mathrm{d} \varepsilon}\right)_{I}\left(\varepsilon^{V}-\varepsilon^{I}\right)+\frac{1}{2}\left(\frac{\mathrm{d}^{2} \phi}{\mathrm{d} \varepsilon^{2}}\right)_{I}\left(\varepsilon^{V}-\varepsilon^{I}\right)^{2}
$$

where

$$
(\mathrm{d} \phi / \mathrm{d} \varepsilon)_{l}=\mathscr{T}^{I}
$$

is the cable tension in terms of Kirchhoff stress, which is related to the actual cable tension in $\mathscr{C}^{l}$ by the equation 


$$
\mathscr{T}^{I}=T^{I}\left(\mathrm{~d} s^{\circ} / \mathrm{d} s^{I}\right)
$$

and

$$
\left(\mathrm{d}^{2} \phi / \mathrm{d} \varepsilon^{2}\right)_{I}=k^{\circ}=E A
$$

is the axial rigidity of the cable.

By means of equations (3), (6), (7) and (8), equation (5) is written in terms of quantities referred to the initial state

$$
\phi^{V}=\phi^{l}+T^{I}\left(\mathrm{~d} s^{I} / \mathrm{d} s^{\circ}\right) \varepsilon+1 / 2 k^{*}\left(\mathrm{~d} s^{I} / \mathrm{d} s^{\circ}\right) \varepsilon^{2}
$$

where

$$
k^{*}=k^{\circ}\left(\mathrm{d} s^{l} / \mathrm{d} s^{\circ}\right)^{3} .
$$

The equations of motion of the cable are obtained using Hamilton's principle which reads

$$
\delta H=\int_{t_{1}}^{t_{2}}\left(\delta K^{v}-\delta \Pi^{v}+\delta W^{v}\right) \mathrm{d} t=0
$$

where

$$
\begin{gathered}
K^{V}\left(\dot{u}_{i}\right)=1 / 2 \int_{l_{c}^{l}} m^{I} \dot{u}_{i} \dot{u}_{i} \mathrm{~d} s^{I} \\
\Pi^{V}(\varepsilon)=\int_{l_{c}^{\circ}} \phi^{V} \mathrm{~d} s^{\circ} \\
W^{V}\left(u_{i}\right)=W^{I}+\int_{l_{c}^{I}} m^{I} g u_{2} \mathrm{~d} s^{I}
\end{gathered}
$$

are the total kinetic and potential energy in $\mathscr{C}^{V}$ and the work done by the cable dead load; the conservation of mass states that $m^{V}=m^{I}\left(\mathrm{~d} s^{l} / \mathrm{d} s^{V}\right)$.

Use of equations (9)-(14), integrating by parts and accounting for the static equilibrium configuration $\mathscr{C}^{1}$ leads to the equations of motion

$$
\frac{\partial}{\partial s^{I}}\left\{T^{I} \frac{\partial u_{i}}{\partial s^{I}}+k^{*}\left(\frac{\mathrm{d} x_{i}^{I}}{\mathrm{~d} s^{I}}+\frac{\hat{c} u_{i}}{\partial s^{I}}\right)\left(\frac{\mathrm{d} x_{j}^{I}}{\mathrm{~d} s^{I}} \frac{\partial u_{j}}{\partial s^{I}}+\frac{1}{2} \frac{\partial u_{j}}{\partial s^{I}} \frac{\partial u_{j}}{\partial s^{I}}\right)\right\}=m^{I} \ddot{u}_{i}
$$

which contain quadratic and cubic non-linearities in the derivatives of the displacement components.

As far as the cable tension in $\mathscr{C}^{V}$ is concerned, equation (5) leads to

$$
\mathscr{T}^{V}=(\mathrm{d} \phi / \mathrm{d} \varepsilon)_{V}=(\mathrm{d} \phi / \mathrm{d} \varepsilon)_{I}+\left(d^{2} \phi / \mathrm{d} \varepsilon^{2}\right)_{I}\left(\varepsilon^{V}-\varepsilon^{I}\right)
$$

and thus the actual cable tension follows

$$
T^{v}=\mathscr{T}^{v}\left(\mathrm{~d} s^{v} / \mathrm{d} s^{\circ}\right)=\left(T^{I}+k^{*} \varepsilon\right)\left(\mathrm{d} s^{v} / \mathrm{d} s^{I}\right) .
$$

In order to obtain an analytical solution to the problem of free inplane vibrations of the cable a simpler form of the equations of motion is deduced by introducing the following assumptions:

the static equilibrium configuration is represented through the parabolic profile:

$$
y=4 d\left[x / l-(x / l)^{2}\right]
$$


which entails $\mathrm{d} s^{I} \simeq \mathrm{d} x^{I}$ and $T^{I} \simeq H, H$ being the horizontal component of the initial tension;

the initial strain is negligible with respect to unity, which corresponds to assume $k^{*}=k^{\circ}$ and $H / E A \ll 1$;

the gradient of the horizontal component of the dynamic displacement is negligible as well (moderately large rotations in $\mathscr{C}^{I} \rightarrow \mathscr{C}^{V}$ ), which corresponds to measure the strain according to Biot.

By omitting the superscript $I$, we find

$$
\begin{gathered}
\frac{\partial}{\partial x}\left\{E A\left[\frac{\partial u}{\partial x}+\frac{\mathrm{d} y}{\mathrm{~d} x} \frac{\partial v}{\partial x}+\frac{1}{2}\left(\frac{\partial v}{\partial x}\right)^{2}\right]\right\}=m \ddot{u} \\
\frac{\partial}{\partial x}\left\{H \frac{\partial v}{\partial x}+E A\left(\frac{\mathrm{d} y}{\mathrm{~d} x}+\frac{\partial v}{\partial x}\right)\left[\frac{\partial u}{\partial x}+\frac{\mathrm{d} y}{\mathrm{~d} x} \frac{\partial v}{\partial x}+\frac{1}{2}\left(\frac{\partial v}{\partial x}\right)^{2}\right]\right\}=m i
\end{gathered}
$$

The bracketed expression \{\} in the first of equations (19) is the additional tension $h=H^{V}-H$ in the cable.

For actual suspended cables the sag-to-span ratio $d / l$ is about $1 / 20, H / E A$ is of order $O\left[(d / l)^{2}\right]$ and the dynamical displacement components are respectively of order $u=O\left(\varepsilon d^{2} / l\right)$ and $v=O(\varepsilon d)$ where $\varepsilon$ is a small parameter of the order of the amplitude; the motion of such cables is correctly described by equations (19) in which terms of order up to $O\left[(d / l)^{3}\right]$ result consistently.

It is possible to reduce the differential problem to one equation only which accounts approximately for both displacement components. The longitudinal inertia forces mü can be neglected in equation (19a) giving

$$
(\partial u / \hat{c} x)+(\mathrm{d} y / \mathrm{d} x)(\partial v / \partial x)+1 / 2(\hat{c} v / \hat{c} x)^{2}=e(t)
$$

according to which the additional cable tension $h=E A e(t)$ is a function of time only. It follows that

$$
u(x, t)=e(t) x-\int_{0}^{x}\left[(\mathrm{~d} y / \mathrm{d} x)(\hat{c} v / \hat{c} x)+(1 / 2)(\partial v / \hat{\partial} x)^{2}\right] \mathrm{d} x
$$

where the value of the constant $e(t)$ is determined through the boundary condition $u(l, t)=0$

$$
e(t)=(1 / l) \int_{0}^{l}\left[(\mathrm{~d} y / \mathrm{d} x)(\hat{c} v / \hat{c} x)+(1 / 2)(\hat{c} v / \partial x)^{2}\right] \mathrm{d} x .
$$

Accounting for equations (20) and (22), the motion is described by the unique partial integrodifferential equation in the transverse displacement component

$$
\frac{\partial}{\partial x}\left\{H \frac{\partial v}{\partial x}+\frac{E A}{l}\left(\frac{\mathrm{d} y}{\mathrm{~d} x}+\frac{\partial v}{\partial x}\right) \int_{0}^{l}\left[\frac{\mathrm{d} y}{\mathrm{~d} x} \frac{\partial v}{\partial x}+\frac{1}{2}\left(\frac{\partial v}{\partial x}\right)^{2}\right] \mathrm{d} x\right\}=m i
$$

The technique used for eliminating the horizontal component is the same adopted in the nonlinear study of the shallow arches [1] and of the taut string [14], the equation of the latter in fact being obtained from equation (23) for $y(x) \equiv 0$; in the linear field it was used in the study of suspended cables [12].

It is convenient to obtain a nondimensional form of the equation of motion. The following positions are introduced

$$
\mu=E A / H \quad v=d / l \quad \tilde{x}=x / l \quad \tilde{t}=\omega t
$$

where $\omega$ is the linear frequency of a generic eigenmode of the cable; the corresponding frequency of the taut string with the same tension $H$ is 


$$
\omega_{0}=n \frac{\pi}{l} \sqrt{\frac{H}{m}} \quad(n=1,2,3 \ldots) .
$$

Two different nondimensionalizations are considered for the displacement components, one with respect to the cable span, the other to the initial sag; in the two cases the equation of motion reads, omitting the tilde

(a) $\tilde{u}=u / l$ and $\tilde{v}=v / l$

$$
\begin{aligned}
\frac{\hat{c}}{\hat{c} x}\left\{\frac{\partial v}{\partial x}+\mu\left[8 v\left(\frac{1}{2}-x\right)+\frac{\partial v}{\partial x}\right] \int_{0}^{1}\left[8 v\left(\frac{1}{2}-x\right) \frac{\partial v}{\partial x}+\frac{1}{2}\left(\frac{\partial v}{\partial x}\right)^{2}\right] \mathrm{d} x\right\} & = \\
& =\pi^{2} n^{2}\left(\frac{\omega}{\omega_{0}}\right)^{2} \ddot{v}
\end{aligned}
$$

(b) $\bar{u}=u / d$ and $\tilde{i}=v / d$

$$
\begin{aligned}
\frac{\partial}{\partial x}\left\{\frac{\partial v}{\partial x}+\mu\left[8 v\left(\frac{1}{2}-x\right)+v \frac{\partial v}{\partial x}\right] \int_{0}^{1}\left[8 v\left(\frac{1}{2}-x\right) \frac{\partial v}{\partial x}+\frac{1}{2} v\left(\frac{\partial v}{\partial x}\right)^{2}\right] \mathrm{d} x\right\} & = \\
& =\pi^{2} n^{2}\left(\frac{\omega}{\omega_{0}}\right)^{2} i
\end{aligned}
$$

where the dot denotes the derivative with respect to $t$.

In the following numerical analysis reference will be made either to equation (26) or to equation (27), each nondimensionalization having its own advantage in the description of the results. Here it is worth noticing that the nondimensionalization with respect to span still furnishes the non-linear equation of the taut string as $v \rightarrow 0$, while that with respect to sag fails in this case.

\section{DISCRETE MODEL AND PERTURBATIONAL SOLUTION}

The study of the influence of the non-linear terms on a vibration mode is developed by assuming a deflection shape of the cable constant during the motion and given by the eigenfuaction $f(x)$ of the linearized equation (23) corresponding to the natural frequency $\omega$. For both symmetric and antisymmetric modes, $\omega$ and $f(x)$ are given in [12], where it is shown that the symmetric linear vibrations of the cable are governed by the unique parameter $\lambda^{2}$ collecting its geometrical and mechanical properties; when $v \ll 1$ it is $\lambda^{2}=64 \mu v^{2}$.

The transverse displacement component of the cable in the nonlinear free motion is represented through separate variables, as follows

$$
v(x, t)=f(x) q(t)
$$

$q(t)$ describing the temporal behaviour.

By applying the Galerkin method to equation (23), one ordinary differential equation is obtained

$$
\ddot{q}+q+c_{2} q^{2}+c_{3} q^{3}=0
$$

in which the coefficient of the linear term is equal to unity since time is nondimensionalized with respect to the linear cable frequency. For the two nondimensionalizations considered with respect to span or to sag, the coefficients $c_{2}$ and $c_{3}$ of the quadratic and cubic terms have the expressions 
(a) $\quad c_{2}=\frac{12}{\pi^{2}} \mu \nu\left(\frac{\omega_{0}}{\omega}\right)^{2} \frac{I_{2}}{I_{m}} \quad c_{3}=\frac{1}{2 \pi^{2}} \mu\left(\frac{\omega_{0}}{\omega}\right)^{2} \frac{I_{3}}{I_{m}}$

(b) $c_{2}=\frac{12}{\pi^{2}} \mu v^{2}\left(\frac{\omega_{0}}{\omega}\right)^{2} \frac{I_{2}}{I_{m}} \quad c_{3}=\frac{1}{2 \pi^{2}} \mu v^{2}\left(\frac{\omega_{0}}{\omega}\right)^{2} \frac{I_{3}}{I_{m}}$

where the following abbreviations are used

$$
I_{2}=\int_{0}^{1}(\mathrm{~d} f / \mathrm{d} x)^{2} \mathrm{~d} x \int_{0}^{1} f \mathrm{~d} x, I_{3}=\left[\int_{0}^{1}(\mathrm{~d} f / \mathrm{d} x)^{2} \mathrm{~d} x\right]^{2}, I_{m}=\int_{0}^{1} f^{2} \mathrm{~d} x .
$$

Bearing in mind that $\omega / \omega_{0}$ and $f(x)$ depend on $\lambda^{2}$ only for the symmetric modes while for the antisymmetric ones $\omega / \omega_{0}$ is equal unity and $f(x)$ is represented by simple sine functions, relations (30) and (31) show that in case (a) the non-linear oscillations of the cable must be described referring separately to the two parameters $\mu$ and $v$ which account respectively for the mechanical and geometrical properties; instead in case (b) reference can be made just to the unique parameter $\lambda^{2}$ which governs the linear dynamics.

An approximate solution to the non-linear equation (29) is pursued through a perturbational procedure; closed-form expressions for the frequency-amplitude relationship and the law of the motion are thus obtained.

By assuming

$$
q=\varepsilon \tilde{q}
$$

where $\varepsilon$ is a small but finite parameter of the order of the motion amplitude and the variable $q$ is of order $O(1)$, equation (29) reads, by omitting the tilde for the sake of simplicity

$$
\ddot{q}+q+\varepsilon c_{2} q^{2}+\varepsilon^{2} c_{3} q^{3}=0 .
$$

Following the method of Lindstedt-Poincarè [14], $q$ is expanded in power series of $\varepsilon$ up to the fourth order term

$$
q=q_{0}+\varepsilon q_{1}+\varepsilon^{2} q_{2}+\varepsilon^{3} q_{3}+\varepsilon^{4} q_{4}+0\left(\varepsilon^{5}\right)
$$

and a strained time coordinate $(\tau)$ is introduced

$$
\tau=\left(1+\varepsilon \alpha_{1}+\varepsilon^{2} \alpha_{2}+\varepsilon^{3} \alpha_{3}+\varepsilon^{4} \alpha_{4}\right) t+0\left(\varepsilon^{3} \eta\right)
$$

Thus, a relation between the non-linear frequency $\Omega$ and the linear one $\omega$ is established

$$
\Omega / \omega=\left(1+\varepsilon \alpha_{1}+\varepsilon^{2} \alpha_{2}+\varepsilon^{3} \alpha_{3}+\varepsilon^{4} \alpha_{4}\right)+0\left(\varepsilon^{5}\right)
$$

By introducing expansions (35) and (36) into equation (34) and equating terms of equal power of $\varepsilon$, the non-linear problem is reconducted to a sequence of linearized equations in each of which the non-linear terms are known from the solutions of the equations of lower order

$$
\left\{\begin{aligned}
\ddot{q}_{0}+q_{0}= & 0 \\
\ddot{q}_{1}+q_{1}= & -2 \alpha_{1} \ddot{q}_{0}-c_{2} q_{0}^{2} \\
\ddot{q}_{2}+q_{2}= & -\left(\alpha_{1}^{2}+2 \alpha_{2}\right) \ddot{q}_{0}-2 \alpha_{1} \ddot{q}_{1}-2 c_{2} q_{0} q_{1}-c_{3} q_{0}^{3} \\
\ddot{q}_{3}+q_{3}= & -2\left(\alpha_{1} \alpha_{2}+\alpha_{3}\right) \ddot{q}_{0}-\left(\alpha_{1}^{2}+2 \alpha_{2}\right) \ddot{q}_{1}-2 \alpha_{1} \ddot{q}_{2}-c_{2} q_{1}^{2}-2 c_{2} q_{0} q_{2}+ \\
& -3 c_{3} q_{0}^{2} q_{1} \\
\ddot{q}_{4}+q_{4}= & -\left(2 \alpha_{4}+2 \alpha_{1} \alpha_{3}+\alpha_{2}^{2}\right) \ddot{q}_{0}-2\left(\alpha_{1} \alpha_{2}+\alpha_{3}\right) \ddot{q}_{1}-\left(\alpha_{1}^{2}+2 \alpha_{2}\right) \ddot{q}_{2}+ \\
& -2 \alpha_{1} \ddot{q}_{3}-2 c_{2} q_{0} q_{3}-2 c_{2} q_{1} q_{2}-3 c_{3} q_{0} q_{1}^{2}-3 c_{3} q_{0}^{2} q_{2} .
\end{aligned}\right.
$$


The dot now denotes the derivative with respect to $\tau$. The imposition of the initial conditions:

$$
q(0)=\bar{q} \quad \dot{q}(0)=\bar{q}
$$

to the unknown variable (35) completes the differential problem.

The solution of equation (38a) (generating solution) reads

$$
q_{0}=A e^{i \tau}+c . c .
$$

where

$$
A=(\tilde{a} / 2) e^{i \beta}
$$

with $\tilde{a}$ and $\beta$ amplitude and phase of the oscillation and c.c. stands for complex conjugate.

Accounting for the generating solution, equation $(38 \mathrm{~b})$ reads

$$
\ddot{q}_{1}+q_{1}=2 \alpha_{1} A e^{i \tau}-c_{2}\left(A^{2} e^{2 i \tau}+A \bar{A}\right)+c . c .
$$

For the series expansion (35) to be valid for times up to $0\left(\varepsilon^{-4}\right)$ (uniformly valid), secular terms like $\tau \cos \tau$ must be eliminated from the solution $q_{1}$; this entails

$$
\alpha_{1}=0
$$

Omitting to introduce the homogeneous solution, equation (42) has the solution

$$
q_{1}=(1 / 3) c_{2} A^{2} e^{2 i \tau}-c_{2} A \bar{A}+c . c .
$$

It is thus noticed that at the $\varepsilon$-order no frequency correction occurs, the solution contains a harmonic of double frequency and a constant term, according to which the midpoint of the motion is drifted with respect to the initial configuration.

Substituting $q_{0}$ and $q_{1}$ in equation (38c), accounting for equation (43) and setting to zero the secular terms gives

$$
\alpha_{2}=\left(\frac{3}{2} c_{3}-\frac{5}{3} c_{2}^{2}\right) A \bar{A}
$$

after which the solution of the equation is determined

$$
q_{2}=\left(\frac{1}{12} c_{2}^{2}+\frac{1}{8} c_{3}\right) A^{3} e^{3 i \tau}+c . c .
$$

A correction of frequency furnished by equation (45) thus occurs at the $\varepsilon^{2}$-order. By proceeding in the same way it follows that

order $\varepsilon^{3}$

$$
\begin{aligned}
\alpha_{3}=0 & \\
q_{3}=\left(\frac{1}{54} c_{2}^{3}+\frac{1}{12} c_{2} c_{3}\right) A^{4} e^{4 i \tau}+\left(-\frac{31}{12} c_{2} c_{3}\right. & \left.+\frac{59}{54} c_{2}^{3}\right) A^{3} \bar{A} e^{2 i \tau} \\
& +\left(5 c_{2} c_{3}-\frac{19}{9} c_{2}^{3}\right) A^{2} \bar{A}^{2}+\text { c.c. }
\end{aligned}
$$

order $\varepsilon^{4}$

$$
\alpha_{4}=\left(-\frac{15}{16} c_{3}^{2}-\frac{485}{108} c_{2}^{4}+\frac{173}{12} c_{3} c_{2}^{2}\right) A^{2} \bar{A}^{2}
$$


By introducing the polar form (41) and accounting for equations (33), (35), (37), (40) and (43)-(49), the following closed-form expressions are obtained for the non-linear frequency of the motion

$$
\Omega=\omega\left\{1+\left[\frac{3}{8} c_{3}-\frac{5}{12} c_{2}^{2}\right] a^{2}+\left[-\frac{15}{256} c_{3}^{2}-\frac{485}{1728} c_{2}^{4}+\frac{173}{192} c_{3} c_{2}^{2}\right] a^{4}\right\}
$$

and the solution

$$
\begin{aligned}
q=a & \cos (\Omega t+\beta)+a^{2}\left\{\frac{c_{2}}{6} \cos 2(\Omega t+\beta)-\frac{c_{2}}{2}\right\}+a^{3}\left(\frac{c_{2}^{2}}{48}+\frac{c_{3}}{32}\right) \cos 3(\Omega t+\beta) \\
+ & a^{4}\left\{\left(-\frac{31}{96} c_{2} c_{3}+\frac{59}{432} c_{2}^{3}\right) \cos 2(\Omega t+\beta)+\left(\frac{1}{432} c_{2}^{3}+\frac{1}{96} c_{2} c_{3}\right) \cos 4(\Omega t+\beta)\right. \\
& \left.+\left(\frac{5}{8} c_{2} c_{3}-\frac{19}{72} c_{2}^{3}\right)\right\}
\end{aligned}
$$

at the unimproved $\varepsilon^{4}$-order, where this wording implies that the corresponding equation was not solved. Therefore the dependence of the non-linear frequency on the fourth power of the amplitude is obtained instead of that on the second power only considered till now when studying curved elements $[1,8,9]$. In the relations $(50)$ and $(51)$ the actual time scale $t$ appears and $a$ is the actual nondimensional amplitude of the motion.

\section{NUMERICAL INVESTIGATION}

The numerical investigation was performed making reference to the motion of the cable in which the deflection shape is represented by the first symmetric mode; indeed this is the first mode of vibration of a cable which exhibits a sag-to-span ratio up to about $1 / 20$ with technical values of $E A / H$. The corresponding eigenfunction normalized in such a way that the maximum amplitude of the vertical displacement component is equal unity and being $v^{2} \ll 1$ reads

$$
f(x)=\frac{\cos \pi \omega / 2 \omega_{0}}{\left(\cos \pi \omega / 2 \omega_{0}\right)-1}\left\{1-\tan \pi \omega / 2 \omega_{0} \sin \left(\pi \omega / \omega_{0}\right) x-\cos \left(\pi \omega / \omega_{0}\right) x\right\}
$$

The ratio $\omega / \omega_{0}$ is furnished in [12] in terms of $\lambda^{2}$.

Attention is focused on the frequency-amplitude relationship of the non-linear motion. This relation, described by the order-four equation (50), is plotted in Fig. 2 referring to the non-dimensionalization with respect to sag for its major effectiveness, by varying the unique parameter $\lambda^{2}$ which governs the dynamic phenomenon in this case. The maximum value of $\lambda^{2}$ considered corresponds to the upper value for prestressed cables, which have positive tension at every amplitude. The corrections of frequency of all cables in this range, from nearly the taut string up to a sag-to-span ratio of about $1 / 20$ for technical values of mechanical properties, can be derived from this figure. It is seen that the behaviour of the cable is initially hardening for low $\lambda^{2}$ values due to the definite prevailing of cubic non-linearities. As $\lambda^{2}$ increases the behaviour is softening at low vibration amplitudes and becomes hardening again as the amplitude increases, according to the behaviour of systems showing both quadratic and cubic non-linearities.

The temporal law of motion in a period for two cables is plotted in Fig. 3 for three values of the amplitude. At the lowest value the law is practically coincident with the cosine function for both cables. As the amplitude increases its shape differs from the linearized solution as stronger as the greater $\lambda^{2}$ is; for cable 6 a drift of the midpoint of oscillation in the upward direction comes out clearly. This phenomenon is illustrated in Fig. 4, in which the upward and downward maximum displacements are shown vs the linear amplitude of oscillation for several cables. For nearly taut cables the drift $b$ is very small since it is essentially due to the 


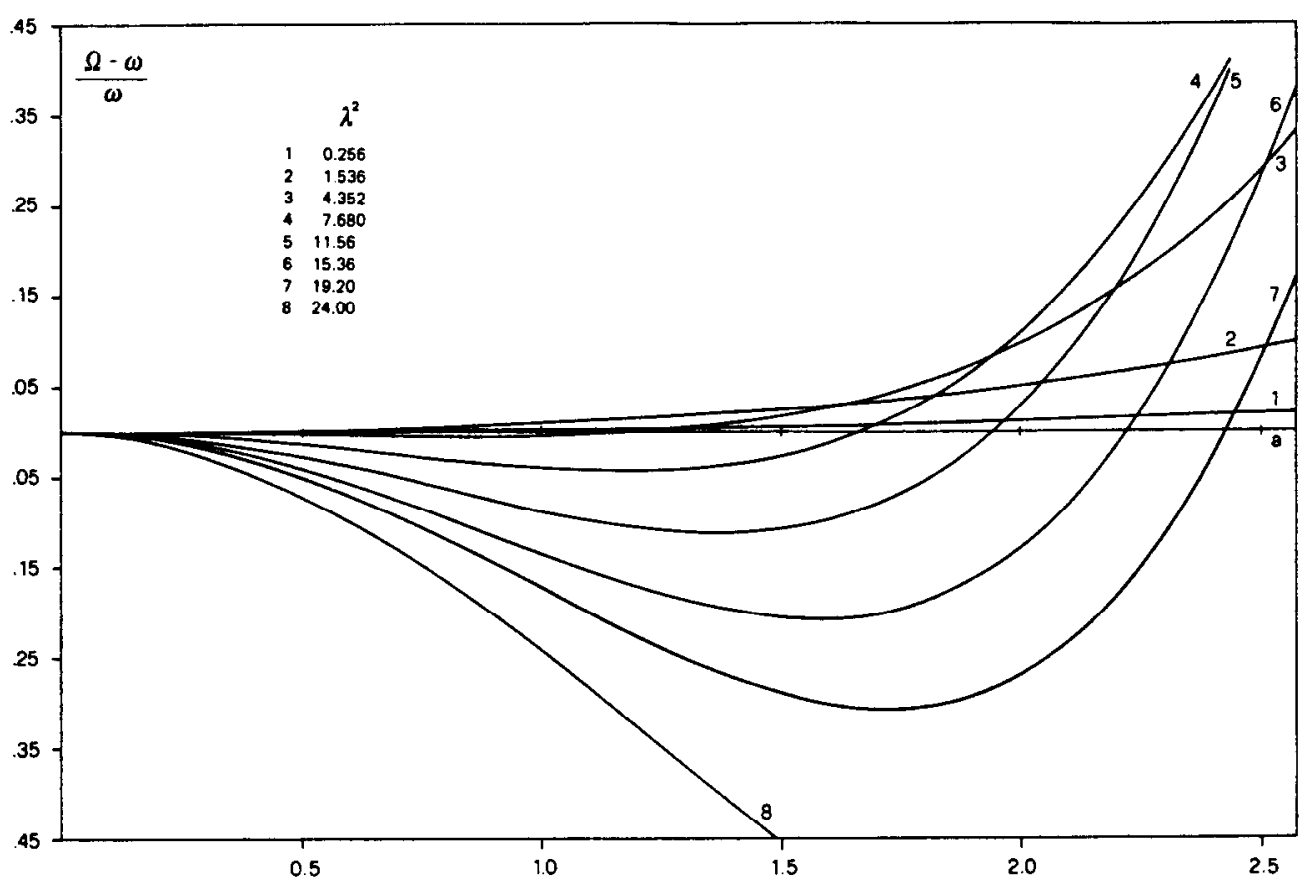

Fig. 2. Frequency-amplitude relationship for different cables: nondimensionalization with respect to sag.

quadratic term; but it becomes quite important for more slack cables and for increasing oscillation amplitudes.

The nondimensionalization with respect to sag does not always give a good description of the dynamic phenomenon; for instance as $d / l$ tends to zero the curves lean on the horizontal axis though the resulting frequency corrections corresponding to absolute amplitude values are high. From this point of view a better representation is furnished by referring to the nondimensionalization with respect to span; in this case however the phenomenon is governed by $E A / H$ and $d / /$ separately and thus an exhaustive description of the problem

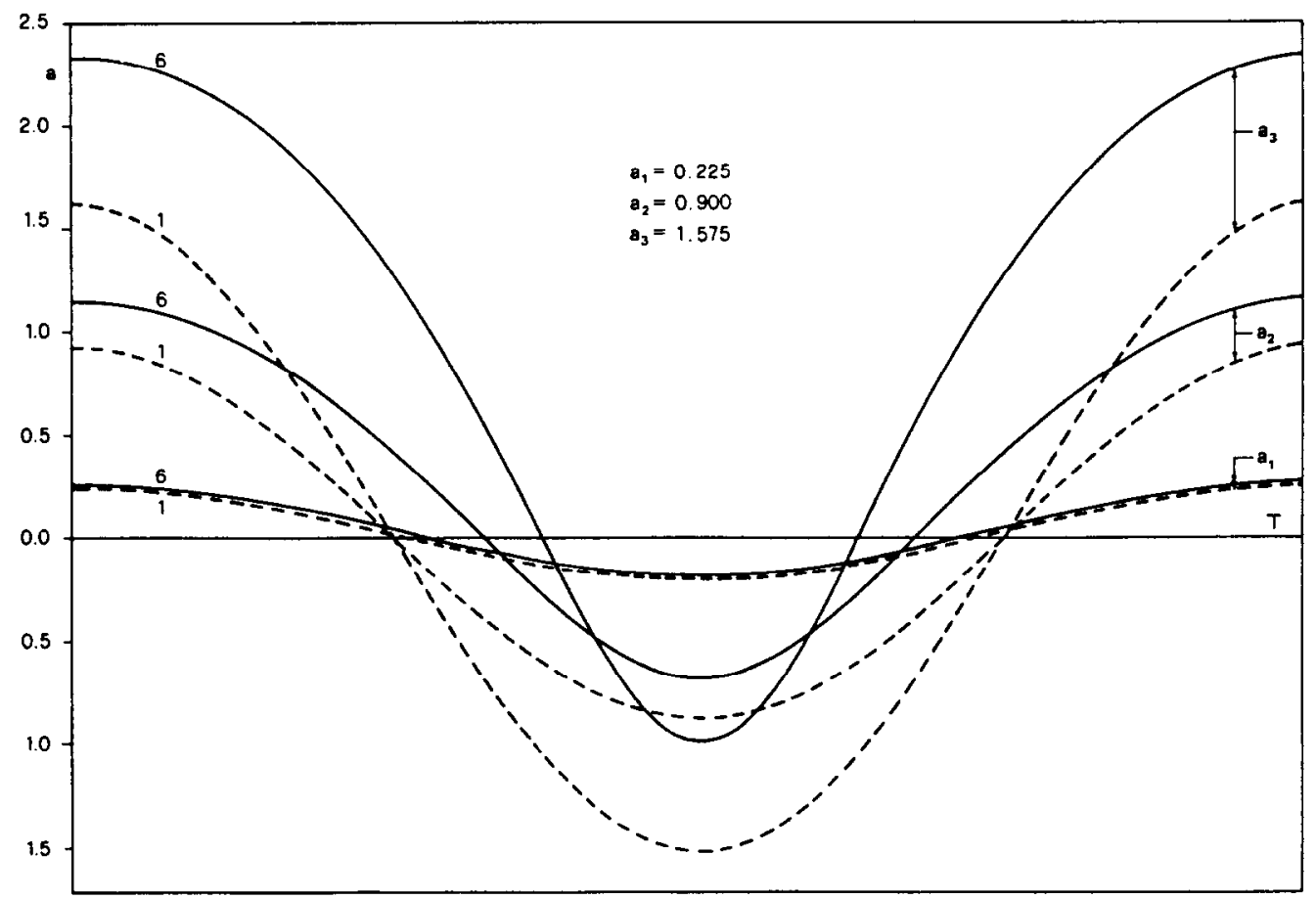

Fig. 3. Law of the motion for cables 1 and 6. 


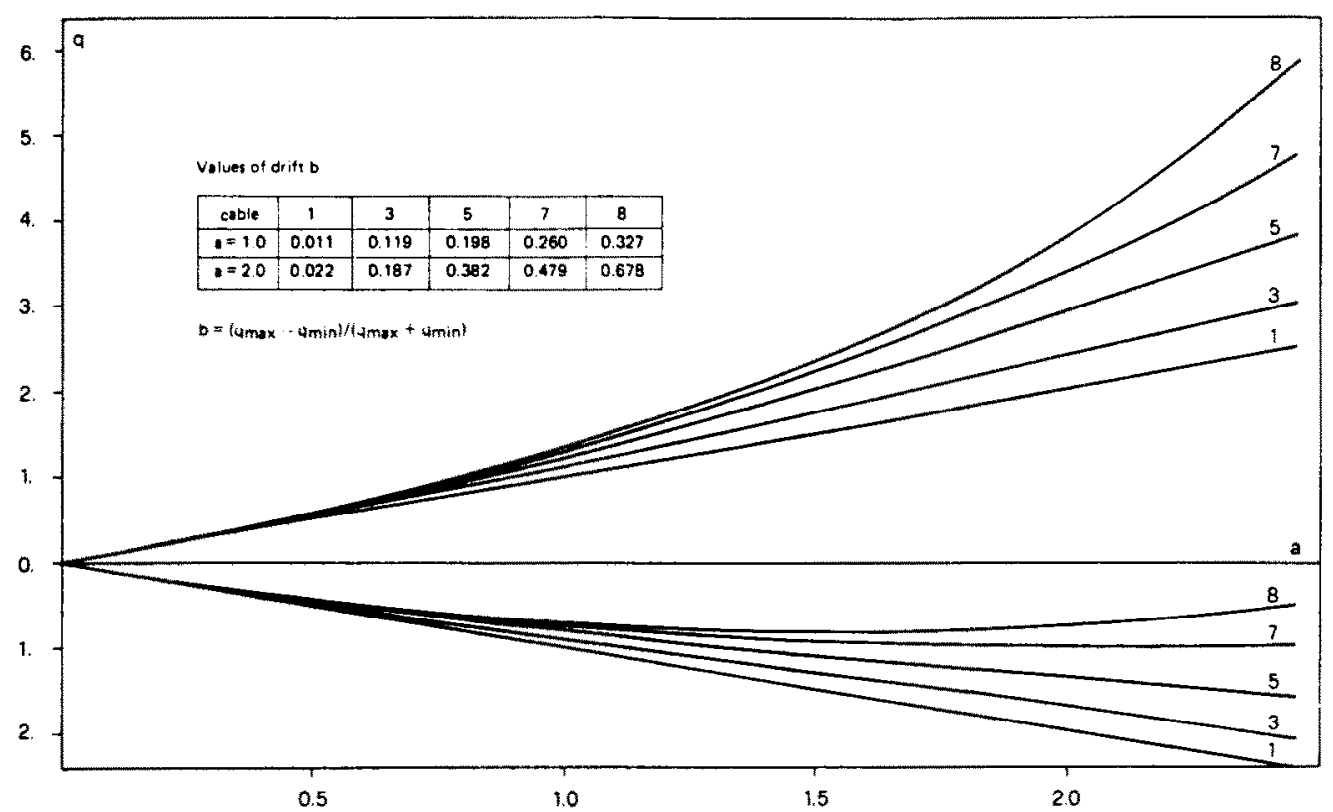

Fig. 4. Maximum upward and downward displacements; percent drift $b$ of the midpoint of oscillation.

requires different charts for prescribed values of one of the two parameters. In Fig. 5 the corrections obtained for two values of $E A / H$ by varying $d / l$ are reported, the five curves plotted for each $E A / H$ corresponding to the $\lambda^{2}$ values of the curves $1,4,5,6,8$ in Fig. 2. Starting from curve 1 ( $\sim$ taut string) which has an always positive correction, as $d / l$ increases each curve exhibits both softening and hardening behaviour depending on the amplitude. The general trend of the curves is very similar to that obtained numerically for shallow arches [2]; in particular a transition amplitude from the softening type of behaviour to the hardening type is evidenced, which increases with $d / l$. The comparison of the results obtained for the two values of $E A / H$ shows that higher non-linearity occurs as higher $E A / H$ is.

The accuracy of the perturbational frequency-amplitude relation is analyzed in Fig. 6 (nondimensionalization with respect to sag) through comparison with the results obtained

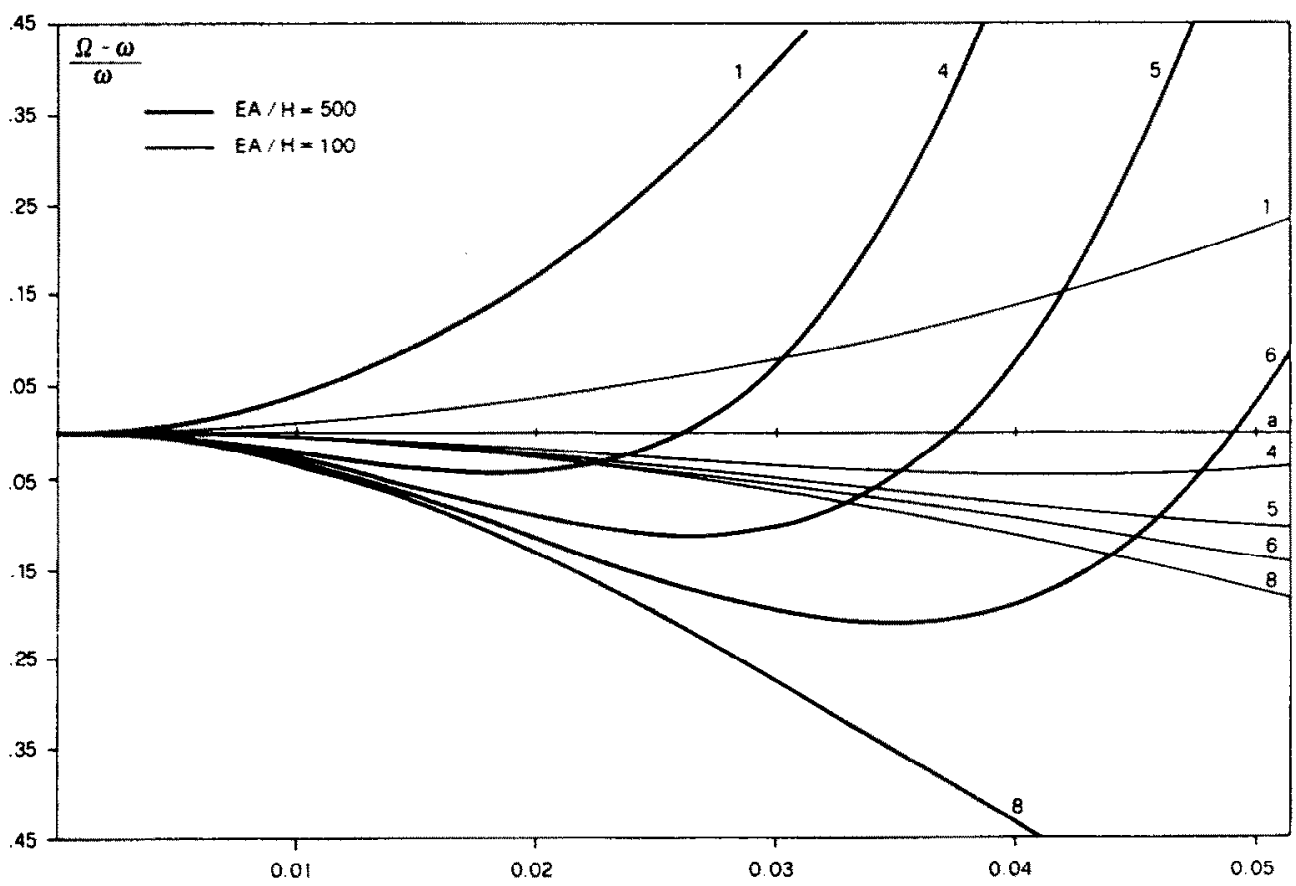

Fig. 5. Frequency-amplitude relationship for different cables: nondimensionalization with respeci to span. 


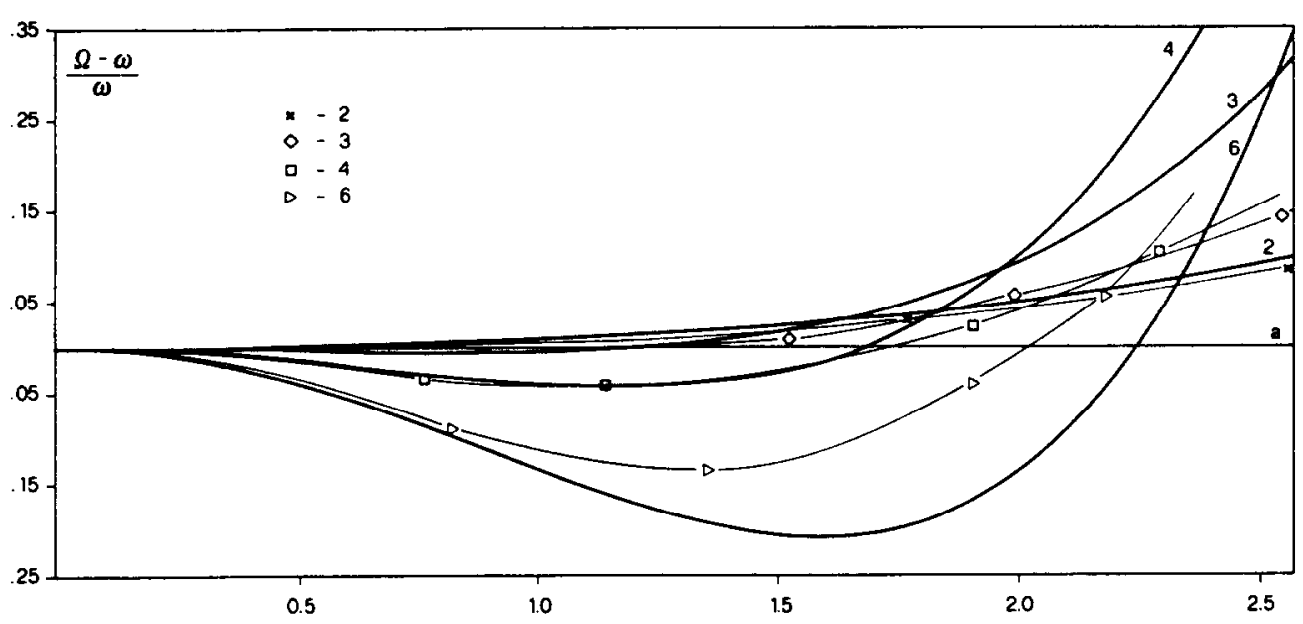

Fig. 6. Comparison between perturbational (thick line) and numerical (thin line) frequency-amplitude relationships.

via a numerical integration of equation of motion (29). It is seen that a perturbation solution - when retaining terms up to the fourth order in the asymptotic expansion - is capable of describing correctly the behaviour of all cables and in particular to show the occurrence of a transition amplitude. In any case however a limited range of amplitudes should be considered for each curve if the error must be taken under a prescribed value.

5. COMMENTS ON A DISCRETE MODEL OBTAINED BY AN ALTERNATIVE PROCEDURE

The problem of planar non-linear oscillations of a cable can be reconducted to the solution of one ordinary differential equation only by means of an alternative procedure followed in the previous works $[8,10]$.

One of the eigenfunctions with two components $g(x)$ and $f(x)$ of the linearized system (19) in which the inertia longitudinal forces are disregarded [12] is assumed as the deflection shape of the cable during the motion. The displacement components are thus expressed as follows

$$
u(x, t)=g(x) q(t) \quad v(x, t)=f(x) q(t) .
$$

Applying the Galerkin procedure to system (19) leads to one non-linear equation similar to equation (29).

The use of the eigenfunction obtained under the assumption above mentioned assumes that

$$
(\hat{c} u / \hat{c} x)+(d y / d x)(\hat{c} v / \hat{c} x)=e(t)
$$

which furnishes an integral relation between the horizontal and vertical components alternative to that given by equations (21) and (22).

This procedure is quite different from the other, namely it establishes a constant relationship between the two displacement components independent of the amplitude of the finite motion. From this point of view the first procedure seems to be the more approximate one, indeed it treats exactly the non-linear relation between $u$ and $v$ in the continuum equation, since the longitudinal inertia forces omitted are actually negligible; so, the approximation is introduced when an assumed shape function only is used to represent the unique variable $v(x, t)$ of the problem. In the second procedure instead the assumption of describing the cable configuration by means of an eigenfunction of the linearized system (19) with two components does not permit exact satisfaction of the non-linear relation (19a), but the two equations are satisfied in the average via an integral method. However, the second procedure still leads to one approximate ordinary differential equation of cable motion which is equivalent to a certain extent to that obtained with the first technique. In particular the 
coefficient $c_{2}$ of the quadratic term is the same in the two cases while the coefficient $c_{3}$ is different because of different definition of the integral $I_{3}$ in equations (30)-(31), which now results

$$
I_{3}=\int_{0}^{1}(\mathrm{~d} f / \mathrm{d} x)^{4} \mathrm{~d} x
$$

For the first symmetric mode the ratio between the coefficients $c_{3}$ obtained in the two discrete models for the nondimensionalization with respect to span is

$$
c_{3}^{H} / c_{3}^{l}=\left(\frac{3}{2}-\frac{2 \sin \pi \omega / \omega_{0}}{\pi \omega / \omega_{0}}+\frac{1}{4} \frac{\sin 2 \pi \omega / \omega_{0}}{\pi \omega / \omega_{0}}\right) /\left(1-\frac{\sin \pi \omega / \omega_{0}}{\pi \omega / \omega_{0}}\right)^{2}
$$

where $c_{3}^{j}$ and $c_{3}^{1 i}$ refer to the former and latter procedure respectively. The values of the ratio $c_{3}^{I I} / c_{3}^{I}$ (see Table 1) show that the second procedure overestimates the correction of frequency in the hardening behaviour; for the taut string it reaches the maximum value equal to 1.5 .

The curves obtained for the frequency-amplitude relationship with the two procedures as the sag increases are compared in Fig. 7, where a finite element solution is also reported. For a more direct evaluation of the approximation of the two procedures, the numerical solutions of the equation of motion (29) are referred to. The finite element results were obtained by discretizing the cable with twenty straight line elements and using the program NONSAP in which the incremental equations of motion are based on an updated Lagrangian formulation [15].

The trend of the sets of curves for the two procedures is similar but considerable differences occur, the curves of the second procedure being more strongly hardening than those relevant to the first. The results obtained with the finite discretization fit closely with those of the first procedure.

To justify the unexpected numerical differences observed, a better insight into the formulation of an approximate discrete model for studying cable oscillations is needed. The

Table 1. Ratio of coefficients $c_{3}$ obtained with the two procedures considered.

\begin{tabular}{ccccccccccc}
\hline$\lambda^{2}$ & 0 & 0.256 & 1.536 & 4.352 & 7.68 & 11.52 & 15.36 & 19.20 & 24.00 & $4 \pi^{2}$ \\
$d_{3}^{1 /} / c_{3}^{j}$ & 1.5000 & 1.4948 & 1.4696 & 1.4196 & 1.3716 & 1.3319 & 1.3103 & 1.3069 & 1.3265 & 1.5000 \\
\hline
\end{tabular}

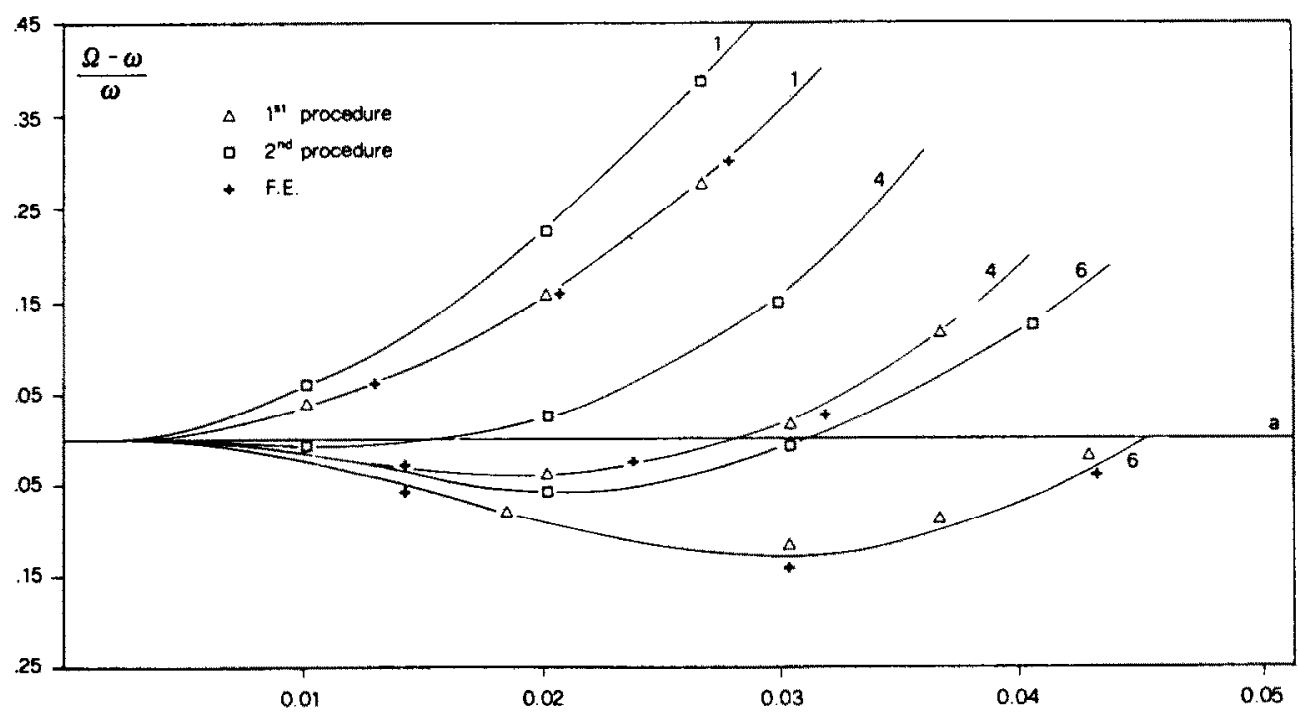

Fig. 7. Comparison of the results obtained with the two procedures considered and with a finite element discretization. 
sequence of linearized equations (38) could be obtained following a different approach, that is by applying the perturbation method to the continuum system (19) at first and then the Galerkin procedure at each order, making use of course of the same shape function. It is useful here to consider this way. By stating

$$
u=\varepsilon u_{0}+\varepsilon^{2} u_{1}+\varepsilon^{3} u_{2} \quad v=\varepsilon v_{0}+\varepsilon^{2} v_{1}+\varepsilon^{3} v_{2}
$$

the following sequence is obtained from system (19), where the prime denotes the derivative with respect to $x$

order $\varepsilon$

$$
\left\{\begin{array}{l}
E A\left(u_{0}^{\prime}+y v_{0}^{\prime}\right)^{\prime}-m \ddot{u}_{0}=0 \\
H v_{0}^{\prime \prime}+E A\left[y^{\prime}\left(u_{0}^{\prime}+y^{\prime} v_{0}^{\prime}\right)\right]^{\prime}-m z_{0}=0
\end{array}\right.
$$

order $\varepsilon^{2}$

$$
\left\{\begin{array}{l}
E A\left(u_{1}^{\prime}+y^{\prime} v_{1}^{\prime}\right)^{\prime}-m \ddot{u}_{1}=-\frac{E A}{2}\left(v_{0}^{\prime 2}\right)^{\prime} \\
H v_{1}^{\prime \prime}+E A\left[y^{\prime}\left(u_{1}^{\prime}+y^{\prime} v_{1}^{\prime}\right)\right]^{\prime}-m \ddot{v}_{1}=-\frac{E A}{2}\left(y^{\prime} v_{0}^{\prime 2}\right)^{\prime}-E A\left[v_{0}^{\prime}\left(u_{0}^{\prime}+y^{\prime} v_{0}^{\prime}\right)\right]^{\prime}
\end{array}\right.
$$

order $\varepsilon^{3}$

$$
\left\{\begin{array}{l}
E A\left(u_{2}^{\prime}+y^{\prime} v_{2}^{\prime}\right)^{\prime}-m \ddot{u}_{2}=-E A\left(v_{0}^{\prime} r_{1}^{\prime}\right)^{\prime} \\
H v_{2}^{\prime \prime}+E A\left[y^{\prime}\left(u_{2}^{\prime}+y^{\prime} v_{2}^{\prime}\right)\right]^{\prime}-m i_{2}^{\prime}=-E A\left[y^{\prime} v_{0}^{\prime} v_{1}^{\prime}+v_{0}^{\prime} u_{1}^{\prime}+u_{0}^{\prime} v_{1}^{\prime}+2 y v_{0}^{\prime} v_{1}^{\prime}+\frac{1}{2} v_{0}^{\prime 3}\right]^{\prime}
\end{array}\right.
$$

Consider the first transversal eigenfunction of system (58), in which $m \ddot{u}_{0}$ is neglected, as the shape function of the cable

$$
u_{0}(x, t)=g(x) q_{0}(t) \quad v_{0}(x, t)=f(x) q_{0}(t)
$$

This eigenfunction is such that

$$
0[g(x)] \simeq 0\left[\frac{d}{l} f(x)\right]
$$

The analysis of system (59) reveals that this shape function is not a suitable one to obtain an approximate solution of that system in which the first equation prevails over the second - in which in particular forced terms vanish as $d / l \rightarrow 0$ - and the order of $u_{1}$ is predicted quite different from $\left(d / / v_{1}\right)$. The use of the eigenfunction $g(x), f(x)$ also as a test function in the Galerkin procedure to satisfy system (59), being given the order of the two components from equation (62), implies underestimating the error associated with equation (59a) and in the limit to neglect it for $d / l=0$, when the integral equation furnishes a vanishing identity. Unfortunately it is important to have a good estimate of $u_{1}$ because the term $v_{0}^{\prime} u_{1}^{\prime}$ in the right side of equation $(60 \mathrm{~b})$ plays an important role in the frequency-amplitude relationship; indeed it is of the same order of the cubic term $v_{0}^{3} / 2$ and both terms are secular.

In substance the second procedure leads to disregard the non-linear relationship between the two components of motion represented by equation (20); this circumstance explains the different results obtained. If this procedure would be followed, at least two different eigenfunctions should be utilized, one of transversal type with a prevailing component $v$ and another of longitudinal type with a prevailing component $u$, in order to satisfy asymptotically the non-linear relationship $u-v$. 


\section{CONCLUSIONS}

Continuum equations of motion of a heavy elastic cable about a deformed initial configuration were developed. Free planar non-linear oscillations are governed or not by a unique parameter depending on the nondimensionalization considered for the displacement variables. Referring to an assumed mode technique, the frequency-amplitude relationship and the law of motion for cables of technical interest were obtained through a perturbation method, which was shown to be capable of describing the actual non-linear phenomenon. More accurate results are obtained if, in reducing the partial differential problem to an ordinary equation, reference is made to one continuum equation of motion after eliminating the horizontal displacement component.

Acknowledgments-This research was partially supported by the Consiglio Nazionale delle Ricerche (Italian Research Council), under Grant N. 82.01453.07.

\section{REFERENCES}

1. L. W. Rehfield, Nonlinear flexural oscillations of shallow arches, AIAA J. 12,91 (1974).

2. G. Prathap and K. A. V. Pandalai, On asymptotic solutions to the nonlinear vibrations of curved elements, $J$. Sound Vibrat. 58, 463 (1978).

3. A. Macwal, Nonlinear fiexural vibrations of an elastic ring. J. appl. Mech. 45, 428 (1978).

4. J. G. Simmonds, Accurate nonlinear equations and a perturbation solution for the free vibrations of a circular elastic ring. J. appl. Mech. 46, 156 (1979).

5. C. C. Glynn and T. L. Maxwell. On the non-linear vibration of a thin ring with steady pressurization. Int. J. NonLinear Mechanics 16. 105 (1981).

6. J. W. Leonard and W. W. Recker, Nonlinear dynamics of cables with low initial tension, J. Engng. Mech. Dit. Am. Soc. Cit: Engrs. 98. 293 (1972).

7. W. W. Carson and A. F. Emery. An energy method determination of large cable dynamics, J.appl. Mech. 43,330 (1976).

8. P. Hagedorn and B. Schäfer, On non-linear free vibrations of an elastic cable. Int. J. Non-Linear Mechanics 15, 333 (1980).

9. A. Luongo, G. Rega and F. Vestroni, Monofrequent oscillations of a non-linear model of a suspended cable, $J$. Sound Vibrat. 82, 247 (1982).

10. G. Rega, F. Vestroni and F. Benedettini, Parametric analysis of large amplitude free vibrations of a suspended cable, Rep. No. 59. Istituto di Scienza delle Costruzioni. Università dell'Aquila (1982).

11. H. Reismann and P. Pawlik, Dynamics of initially stressed hyperelastic solids, SM Archs 2. 129 (1977).

12. H. M. Irvine and T. K. Caughcy, The linear tneory of free vibrations of a suspended cable, Proc. R. Soc. A341.299 (1974).

13. H. M. Irvine, Energy relations for a suspended cable, Q. Jl Mech. appl. Math. 33, 227 (1980).

14. A. H. Nayfeh and D. T. Mook, Nonlinear Oscillations. John Wiley. New York (1979).

15. K. J. Bathe. E. Ramm and E. L. Wilson. Finite element formulations for large deformation dynamic analysis, Int. J. num. Meth. Engng. 9, 353 (1975).

\section{Résumé :}

On développe les équations non linéaires du mouvement libre d'un câble élastique lourd autour d'une configuration initiale déformée. En se référant à une technique de mode supposé on obtient, avec une méthode de Galerkin, une équation ordinaire pour le mouvement plan du câble dont on recherche une solution approchée par une méthode de perturbation. On donne des résultats non dimensionnels appropriés pour les vibratlons du premier mode symétrique avec differentes valeurs des propriétés du câble. On discute quelle est la procédure propre à expliquer logiquement les relations clnématiques non linéaires du câble au moyen d'une équation ordinaire du mouvement.

Zusammenfassung:

Nichtlineare Kontinuumsgleichungen der freien Bewegung eines schweren elastischen Kabels um eine verformte Ausgangsform werden entwickelt. Eine gewoehnliche Gleichung fuer die ebene Bewegung des Kabels wird mit Hilfe eines Galerkinschen Verfahrens aufgestellt, und eine Naeherungsloesung wird durch eine Perturbationsmethode erreicht. Fuer die Schwingungen mit der ersten, symmetrischen schwingungsform und fuer verschiedene Werte der Kabeleigenschaften werden geeignete nichtaimensionale Ergebnisse dargestellt. Es wird untersucht, welches verfahren zuverlaessig die nichtlinearen, kinematischen Beziehungen des Kables in einer gewoehnlichen Bewegungsgleichung gibt. 